\title{
Penser le possible de langue, en linguistique et en poésie
}

\author{
Hérout Raphaëlle \\ LASLAR, Université de Caen Basse-Normandie \\ raphaelle.herout@gmail.com
}

\section{Introduction}

Nous souhaitons dans cet article interroger le rôle que peut jouer la modalité poétique dans la compréhension du «possible de langue », tel qu'il a été défini par Milner (1989), et discuté par Auroux (1991 d). En effet, le concept de possible de langue, qui repose sur le fait que «tout ne peut pas se dire » (Milner 1989 : 55), en introduisant un jugement différentiel, permet de circonscrire les productions jugées possibles ou impossibles en langue. Or la poésie - et nous choisissons ici à dessein le corpus surréaliste tend à explorer ces zones dites impossibles en langue, à " essayer » des constructions atypiques voire agrammaticales, à tester la résistance des structures fermes, dont les emplois véhiculent une norme perçue comme difficilement muable. Il s'agit donc pour nous d'apprécier le point de bascule entre le possible et l'impossible de langue au regard de productions volontairement déviantes, souvent accompagnées d'un discours de justification ou de (pseudo-)rationalisation, ce qui posera inévitablement la question d'un potentiel recul des limites de l'expression, par l'attestation de tournures nouvelles, et la découverte de ressources linguistiques jusqu'alors inobservées voire impensées. Autrement dit, est-il possible, ou non, d'infléchir la raison grammaticale? Et si oui, cela se fait-il au nom d'une recherche explicite, ou simplement au nom d'une intuition créatrice ?

\section{Différentiel positif / négatif}

Ce concept de "possible de langue » s'impose chez Milner au cours d'une réflexion épistémologique d'importance, visant à quitter le paradigme de la science empirique, et à éclairer le débat qui oppose les partisans d'une conception de la linguistique comme science positive aux partisans d'une linguistique qui serait une science normative. C'est la recherche des fondements mêmes de la scientificité de la linguistique qui sous-tend la mise au jour de ce concept, dans son rapport à l'édiction des normes et à l'observation des usages. Abandonner l'approche empirique revient à refuser les "impressions » de grammaticalité ou d'agrammaticalité sur des critères d'observation du type « la donnée de langue X se rencontre [ou ne se rencontre pas] dans l'espace ou dans le temps » (Milner 1989: 50), pour se doter de critères de jugement qui puissent valider des classes et des types d'énoncés.

C'est précisément ce qui fonde la distinction entre possible et impossible de langue : il ne s'agit pas de tester la validité d'énoncés recensés, attestés, (lesquels peuvent être «matériellement possibles » « linguistiquement impossibles ») mais de faire appel à des lois qui président à l'acceptabilité ou non de certains énoncés dans le système de la langue, créant ainsi un raisonnement général à partir de productions isolées. Cette différence entre les énoncés attestés et le jugement différentiel sur ces énoncés est à l'origine de "l'hypothèse grammaticale minimale » qui servira de base à notre réflexion: "le possible de langue et le possible matériel peuvent ne pas coïncider» (Milner 1989: 55). En d'autres termes, rien n'empêche de créer un énoncé (matériellement possible) qui n'ait aucune valeur dans la 
langue donnée (linguistiquement impossible), dans la mesure où « la notion de règle (comme la notion de norme) [...] ne dit pas que ce qui ne suit pas la règle soit irréalisable » (Milner 1989: 87). De la liberté, sans borne aucune, de tout sujet-parlant de produire un fait langagier quel qu'il soit à la reconnaissance de ce fait langagier en tant qu'énoncé raisonnable par la communauté qui le reçoit, nous pouvons observer un continuum allant de l'adéquation du possible matériel et du possible linguistique, à une totale disjonction. De cette disjonction provient justement la possibilité d'une « activité grammaticale » du sujet-parlant, qui émet un jugement différentiel, à partir de sa propre expérience de la langue. Cette activité grammaticale relève de degrés de conscience variables, pouvant aller du sentiment épiliguistique jusqu'au discours métalinguistique, capable de rationaliser les productions langagières. Elle peut également varier à la fois «au sein d'une communauté parlante, fût-elle sociologiquement homogène, [où] peuvent coexister plusieurs systèmes différents de répartition entre formes jugées possibles et impossibles » (Milner 1989: 82), et, à une échelle plus réduite, chez un même individu :

\begin{abstract}
plusieurs grammaires distinctes coexistent [...] parfois chez le même individu. Il arrive alors que le sujet juge une donnée $\mathrm{D}$ suivant les critères d'une grammaire $\mathrm{G}$, alors que cette donnée relève en fait d'une autre grammaire G'. C'est le cas le plus fréquent : ce qu'on appelle usuellement en français une faute de grammaire est en réalité une faute à l'égard d'une seule grammaire (disons: Grevisse), or le français connaît plusieurs grammaires, c'est-à-dire plusieurs répartitions différentielles du possible de langue et de l'impossible de langue. (Milner $1989: 84$ )
\end{abstract}

Les bornes positive et négative, si elles restent bien immuables, semblent alors disposer d'un champ d'attraction qui, lui, peut être plus ou moins puissant ou résistant, qui ferait bouger la frontière du possible de langue, permettant entre autre d'adapter son jugement à des contextes différents et à des postures hétérogènes.

Si cette image nous fait temporairement abandonner les critères rigoureux et finis qui doivent présider à l'analyse linguistique, elle possède néanmoins la vertu de souligner la difficulté, qui se rencontre parfois, d'émettre un jugement véritablement différentiel, autrement dit de trancher sur le caractère possible ou non d'un énoncé.

C'est ce qui ressort de nombreuses études, telle celle de Monique Debièvre ${ }^{1}$, qui exploite les différences d'appréciation d'un même fait linguistique, selon qu'on le juge correct ou non, selon qu'on pourrait le dire ou non, et selon qu'on le comprenne ou non. Bien entendu, des résultats fort différents émanent des trois parties du questionnaire, ce qui montre un degré variable d'acceptation, entre le fait de considérer un énoncé comme correct et le fait de se l'approprier, d'en être l'énonciateur, et donc d'intégrer de l'hétérogénéité linguistique, de l'irrégularité, dans sa propre parole.

\title{
3 Le surréalisme ou les « limites-non frontières » du possible de langue
}

A partir de ces fondements, c'est bien la résistance plus ou moins robuste de certaines structures, volontairement mises à l'épreuve et transgressées, que nous souhaitons soumettre au jugement différentiel évoqué. Interroger le possible de langue revient donc à hiérarchiser les usages et à tenter d'apprécier leur répercussion sur le système de la langue.

Nous choisissons nos exemples dans la poésie surréaliste, qui se distingue par un engagement langagier particulier. En effet, ce mouvement culturel et littéraire s'est pensé, comme le rappelle André Breton, comme une «opération de grande envergure portant sur le langage» (Breton [1955], 2008 : 18). Il s'agissait, par l'action de la Révolution Surréaliste, d'agir sur le monde, de le transformer en bousculant nos représentations, qui perpétuent une pensée et un monde jugés médiocres. Nos représentations étant 
véhiculées par le langage, il était évident de faire de ce langage l'opérateur principal du bouleversement sociétal espéré. Émanciper le langage devait donc permettre d'émanciper l'homme, et d'insuffler de nouvelles valeurs dans une société sclérosée. Tout le travail poétique entrepris devait ainsi permettre de libérer le langage de son usage purement utilitariste, pour en faire un véritable moyen d'expression, de création et de connaissance. Il n'était aucunement question de mettre à mal la langue comme cela s'est fait à l'époque Dada, mais bien de questionner l'accès à la signification, de subvertir l'évidence de la construction du sens et de la communication.

Si une grande part du caractère novateur de l'écriture surréaliste tient à son emploi dépaysant des images et aux associations sémantiques inhabituelles voire déroutantes ${ }^{2}$, nous préférons, dans le cadre de cet article, nous limiter à ce qui touche l'axe syntagmatique du langage, dans la mesure où la perturbation poétique de la syntaxe est un travail autrement plus complexe et où il pose des questions inhérentes au système même de la langue.

\subsection{L'impossible de langue n'est pas (toujours) un défaut de syntaxe}

En guise de précaution, il convient de préciser que s'il y a bien, au sein du surréalisme, une recherche d'une autre façon de dire, cela ne passe pas par le fait de malmener la syntaxe. La question du respect de la syntaxe a été posée très tôt, notamment en réponse aux critiques littéraires qui ne voyaient dans la production d'avant-garde que «manque de syntaxe». Plusieurs textes, parfois à valeur de manifeste, ont été écrits en réaction à ces critiques; citons l'article «Syntaxe», de Pierre Reverdy, publié dans la revue Nord / Sud en avril 1918, ou encore un article portant le même titre, paru en février 1920, signé par Paulhan, dans le premier numéro de la revue Proverbe, qu'il a créée avec Éluard. Ces deux textes sont explicites, à la fois dans le rapport à la norme et aux institutions qui jugent leur production, comme cela apparaît chez Reverdy :

\begin{abstract}
C'est que, ayant pris un modèle et le tenant bien ils [les critiques littéraires] sont surpris de se trouver devant un fait nouveau qui n'a pas de modèle, mais qui, peut-être, en deviendra un. [...] Pour un art nouveau une syntaxe nouvelle était à prévoir ; elle devait fatalement venir mettre dans le nouvel ordre les mots dont nous devions nous servir. [...] La syntaxe est un moyen de création littéraire. C'est une disposition de mots - et une disposition typographique adéquate et légitime.[...] Il faut rendre hommage à ceux qui n'accusent qu'eux de ne pas aimer une chose nouvelle qu'ils ne comprennent pas. Ceux qui, grâce à leur prétention et à leur insensibilité, se contentent de vouloir ramener tout à leurs vieux espoirs, sont détestables.
\end{abstract}

Tout le monde ne peut pas marcher au même pas.

Paulhan émet des commentaires semblables, et l'on en retrouve encore, quelques années plus tard, sous la plume de Breton, qui s'explique ainsi : "Qu'on y prenne garde, je sais le sens de tous mes mots et j'observe naturellement la syntaxe (la syntaxe qui n'est pas, comme le croient certains sots, une discipline). » (Breton [1927] $1992:$ 275)

Une fois prises en compte ces informations liminaires sur l'importance de la syntaxe, à la fois acquise et naturellement respectée, et lieu d'interrogations fondamentales sur le pouvoir-dire, de sorte qu'elle devient objet de manipulations, il convient de « marcher au pas » de ces nouvelles structures syntaxiques pour en discerner le fonctionnement. Comment la langue accueille-t-elle ces structures ? S'agit-il d'investir des zones libres, non encore balisées par la conscience grammaticale, ou de bouleverser des formes devenues classiques ? Et à quel niveau y a-t-il péril pour la signification? 
Pour appréhender ces questions en contexte poétique, nous ne dresserons pas une typologie des écarts normatifs, mais choisirons quelques faits syntaxiques marquants dont on trouve un nombre d'occurrences significatif, afin d'analyser le passage, lorsqu'il survient, de l'impossible au possible de langue et d'apprécier le changement qu'il inscrit dans la langue. Nous partons pour cela du postulat que le texte littéraire est une source pertinente pour le linguiste en ce qu'il fournit un observatoire de la langue, et qu'il est un lieu de concrétisation de potentialités linguistiques non encore attestées.

Ces énoncés qui mettent en question le possible de langue, nous les envisageons, selon la terminologie de Culioli, comme des transformations : " [est appelée] transformation toute opération qui transforme une configuration en une autre » (Culioli, $1990: 128)$.

\section{Possible / impossible et collusion de syntagmes}

Parmi ces transformations possibles, le jeu sur les bornes des syntagmes, en interférant avec le parenthésage régulier des unités, introduit dans la langue quelques perturbations intéressantes à analyser, dont voici deux exemples :

\section{(1) Un jour qu'il fera nuit d'amour ${ }^{3}$}

(2) Bientôt, il va partir, il va sourire d'enfant $\mathbf{t}^{4}$

En (1), nous n'analysons pas l'incompatibilité sémantique apparente entre les sèmes du jour et de la nuit (qu'il suffise, dans un premier temps, de se rappeler que, selon Breton, pour les surréalistes «[t]out porte à croire qu'il existe un certain point de l'esprit d'où la vie et la mort, le réel et l'imaginaire, le passé et le futur, le communicable et l'incommunicable, le haut et le bas cessent d'être perçus contradictoirement. $\left.\rangle^{5}\right)$.

L'intérêt pour nous réside dans la création d'une structure mixte qui comporte à elle seule deux syntagmes de natures différentes, censément distincts dans l'usage normal: [il fait nuit] et [une nuit d'amour]. L'élément nominal commun, tantôt complément au sein d'une locution impersonnelle, tantôt tête nominale d'un syntagme lexicalisé, va se voir doté des deux fonctions, ce qui est, a priori, impossible. On n'est pas là dans le cadre d'une ambiguité syntaxique, mais bien d'une syntaxe plurielle, qui combine plusieurs niveaux d'analyse. Nous pouvons donc lire cet énoncé comme le fruit d'une transformation de la relation prédicative : le verbe reste bien impersonnel, mais le complément connaît une expansion qui le modifie en substance. Ainsi à la locution « faire nuit » se superpose, sous la plume du poète, la locution « faire nuit d'amour » qui, en plus d'être parfaitement évocatrice, modifie l'organisation de l'axe syntagmatique. Il serait en effet possible de représenter ce segment ainsi :

« Un jour qu'il fera nuit

nuit d'amour »

avec le double axe syntagmatique et paradigmatique, où le constituant qui apparaît deux fois va se fondre en une seule occurrence, faisant remonter le deuxième $\mathrm{SN}$ au niveau du premier syntagme verbal (SV).

Cela illustre à la fois un mode de création verbale par enchaînement d'idées (sur le signifié autant que sur le signifiant, puisque c'est bien ce signifiant qui fait la jonction, presque la fusion, entre les deux syntagmes), et un nouveau rapport aux choses puisqu'ainsi inclus dans une locution impersonnelle, l'amour devient un élément de description du monde physique, susceptible de frapper quiconque à 
n'importe quel moment. Ajoutons que d'un point de vue sémantique, la présence de deux éléments rhématiques dans une locution brève crée un effet de densité remarquable.

L'exemple (2) s'analyse de manière semblable, avec un SV [il va sourire], et un SN [un sourire d'enfant] qui se télescopent. Le cas est néanmoins plus complexe dans la mesure où une recatégorisation intervient. Le verbe sourire est normalement intransitif, le seul argument qu'il régit est le sujet. Bien sûr selon les tournures, il peut admettre un complément prépositionnel, comme dans «sourire de bonheur» par exemple, mais dans ce cas, le complément indique le sentiment qui cause, qui est à l'origine du sourire. Peut-on alors «sourire d'enfant» comme on sourirait de joie ? Certes non, mais encore une fois, l'image créée est tout à fait intelligible et semble tirer parti d'un potentiel expressif fondé notamment sur l'isomorphie de la forme infinitive du verbe et du substantif, qui favorise la transformation de l'une en l'autre, et ouvre à différentes expansions.

La superposition du SN [sourire d'enfant] au SV [il va sourire] peut ainsi se lire comme une transformation du SN, qui devient un véritable SV, créant de la sorte une nouvelle relation prédicative, complexe et atypique. Si cette construction est poétiquement intéressante, elle suscite également quelques remarques qui touchent au système même de la langue puisque la recatégorisation opérée provoque une instabilité non-prévisible. En effet la recatégorisation (ou translation selon la terminologie de Tesnière) est possible grâce à une certaine plasticité de la langue : un mot susceptible de remplir, dans un contexte donné, un rôle fonctionnel qui n'est pas le sien dans sa classe initiale est un mot qui va être vecteur d'instabilité dans la langue. En passant d'une catégorie ou d'une classe à une autre, l'élément translaté va faire interférer les parties du discours. Dans l'exemple qui nous intéresse, nous avons deux structures qui sont, prises isolément, tout à fait fermes et à ce titre difficilement soumises à l'instabilité. Or, la reconnaissance première de la séquence auxiliaire + verbe à l'infinitif transforme (ou translate) la séquence nom + complément du nom. Le complément du nom «sourire» devient complément circonstanciel de manière du verbe «sourire » (sourire comme seuls les enfants peuvent sourire), et occupe la seule place disponible qui est une place de complément de cause. Sur ce point, les analyses de Danielle Leeman sur les constructions verbales à complément de cause nous éclairent : "d'une manière générale, les verbes [en $d e+$ complément de cause] désignent le témoignage involontaire [...] d'un sentiment ou d'une sensation soit par un état, soit par un changement d'état, soit par une action dont le sujet est le patient plutôt que l'agent effectif » (1991: 81). Dans le cadre de la poésie surréaliste et du reclassement des valeurs qu'elle propose, cette présence de l'enfance (comme moment privilégié où la pensée et l'imagination n'ont pas encore été bridées par la logique utilitariste) qui acquiert un rôle actif en transformant le sujet en patient est tout à fait signifiante. Sourire d'enfance c'est donc aussi se laisser habiter, ou se voir investi par la puissance de l'enfance, qui se manifeste à l'occasion d'un sourire. La contrainte combinatoire qui pèse sur le choix du complément ainsi transgressée donne à lire, dans l'impossible de langue, une réalité ressentie et exprimée dans de fines nuances. Cet exemple est donc remarquable dans la façon qu'il a de provoquer de l'instabilité là où les formes sont a priori stables, et c'est précisément dans ces zones accidentées que vont se tester de nouveaux possibles de langue.

De ces deux exemples, nous souhaitons retenir le fait qu'ils sont emblématiques d'un "plus-à-dire » par rapport à ce que l'usage commun permet. La compréhension de ces exemples n'est pas très éloignée de celle des mots-valises, sauf que les deux éléments ne sont pas agglomérés. L'analyse qu'en donne Marc Bonhomme nous paraît tout à fait applicable aux constructions qui nous concernent : " le sens du motvalise est supérieur au sens additionné de ses constituants. Et c'est précisément par ce supplément de sens qu'il fait bouger les frontières de notre encyclopédie » (Bonhomme 2009 : 53).

C'est précisément la relation entre les frontières de l'encyclopédie, les représentations qui les sous-tendent et les structures linguistiques qui les portent qui nous intéresse dans l'interprétation de ces exemples qui inventent de nouvelles façons de dire. 


\section{Possible / impossible et contraintes combinatoires}

D'autres exemples, ne mettant cette fois pas en jeu un télescopage de syntagmes, présentent également des agrammaticalités basées sur l'inadéquation entre le verbe et son complément, qui orientent le jugement différentiel vers la borne négative. Plusieurs occurrences construites à partir du verbe savoir apparaissent :

(3) Cette blancheur, enfin, s'avéra au point que je m'étonnais de la savoir ${ }^{6}$.

(4) Le volubilis et je sais l'hypoténuse ${ }^{7}$.

(5) Nous ne savons plus rien que les astres morts ${ }^{8}$

L'exemple (3) articule deux agrammaticalités : la première est sémantique, « savoir la blancheur » relève incontestablement de l'impossible de langue. Pourtant les noms abstraits peuvent tout-à-fait être complément du verbe savoir, comme dans «savoir le pourquoi et le comment », donné en exemple dans le dictionnaire ${ }^{9}$. Le problème, s'il réside bien dans le choix du complément, tient donc plus au fait que le verbe savoir engage, comme l'explique Rémi-Giraud (1986 : 250) une «sorte de connaissance qui parle des choses [où] le sujet reste dans la réalité intérieure d'un processus mental », ce qui s'accommode mal avec l'idée de «blancheur». Et, plus précisément, Franckel et Lebaud (1990) montrent que dans la répartition des usages entre savoir et connaître se joue la différence entre le rapport du sujet à l'existence en soi d'un objet (connaître) et le rapport du sujet aux propriétés de l'objet (savoir). L'emploi de savoir « engendre une dissociation entre la dénomination [de l'objet] et l'énoncé de son contenu, c'est-à-dire de ses déterminations qualitatives» $»(1990$ : 88). Donc dans les exemples en question, savoir «la blancheur », "l'hypoténuse » ou encore " les astres morts », ce n'est pas savoir qu'ils sont, qu'ils existent, mais savoir ce qu'est la blancheur, l'hypoténuse, ce que sont les astres morts. Autrement dit, le complément de savoir relève normalement du fonctionnement prédicatif ; l'anomalie permet ici de rendre prédicatifs des éléments censément non-prédicatifs.

La seconde agrammaticalité s'envisage d'un point de vue syntaxique, et l'analyse est plus complexe ; si l'emploi de savoir avec un pronom est bien régulier (comme dans « je ne veux pas le savoir »), le pronom doit dans ce cas être le substitut d'une proposition (complétive ou interrogative indirecte), et non d'un nom ou d'un SN. Or dans notre exemple, l'antécédent est bien «cette blancheur », ce qui motive la forme féminine du pronom, là où l'usage normal requiert la forme masculine/neutre, en lieu et place d'une proposition. Si le pronom et son antécédent sont parfaitement coréférentiels, et en cela assurent l'intelligibilité du propos, cet emploi n'entre pas dans le champ des constructions possibles du verbe savoir. Mais cette présence peut s'expliquer en prenant en compte une double ambiguïté : tout d'abord, et toujours en considérant savoir dans son emploi verbal (et non en tant qu'auxiliaire modal), l'exemple (3) « réveille » l'empreinte de la construction avec attribut de l'objet, du type « je la sais heureuse » ou « je la sais en danger », mais ne l'actualise pas, puisque cette dernière place possible reste vacante. Il y a donc un effet d'amorçage qui va laisser en suspens l'ombre d'un prédicat et ainsi, par contraste, mettre l'accent sur le sémantisme intrinsèque du verbe savoir, en emploi absolu.

C'est là où intervient l'autre ambiguïté de cet énoncé, car à sa lecture, on est tenté de commuter le verbe savoir avec connaître, qui, lui, admet un complément pronominalisé qui soit un nom ou un SN. En effet, il serait beaucoup plus aisé de lire : «cette blancheur, je la connais », mais ces deux verbes, pour synonymes qu'ils puissent paraître, engagent deux rapports différents à la connaissance. C'est ce qu'explique Jacqueline Picoche (1986 : 128) lorsque, analysant les contraintes liées à ces deux verbes, elle conclut au «caractère essentiellement analytique de la proposition, et [au] caractère synthétique du nom concret. Elle fait ainsi de savoir un verbe d'analyse, et de connaître un verbe de synthèse ». 
Ainsi, l'exemple (3) offre la possibilité d'exprimer de manière analytique un prédicat plutôt synthétique, donc d'appréhender un objet extérieur par un mode de connaissance qui relève plutôt d'une "réalité intérieure ", ce qui est congruent dans la démarche surréaliste d'accroissement de la connaissance intérieure et d'une plus grande adéquation entre la pensée et le monde.

L'exemple (4) se lit de façon similaire, avec ceci de particulier que l'hypoténuse, si elle désigne bien un réalité concrète, est en soi une construction intellectuelle. Il est impossible, en langue, de «savoir l'hypoténuse », à moins, éventuellement, de recourir au sens étymologique («ce qui est tendu sous »), mais cela semble peu probable en contexte d'écriture automatique, et il semble peu judicieux de réduire l'hétérogénéité manifeste de cette construction à un simple emploi étymologique.

L'exemple (5) tire également son impossibilité en langue du fait de l'incompatibilité de construction entre le verbe et son complément, mais la lecture s'opère différemment à cause d'une ambiguïté syntaxique qui perturbe ce qu'il reste de compréhension au-delà de l'agrammaticalité. En effet, "savoir les astres morts » pourrait se lire comme une proposition participiale (que l'on peut gloser par une complétive : nous savons que les astres sont morts), ce qui n'est pas sans conférer un effet d'archaïsme. Mais «morts » peut aussi se lire comme un adjectif qualifiant directement et logiquement «astres », auquel cas on retrouve l'impossibilité analysée en (3), qui pourrait se réduire en commutant savoir avec connaître.

Ces trois exemples nous semblent emblématiques de la recherche, par les poètes surréalistes, d'un certain dépaysement linguistique qui ne tient pas à une complexité de construction, mais au contraire, qui se situe dans les interstices des éléments les plus connus, maîtrisés, et a priori stabilisés dans la langue. Si ces énoncés, à n'en pas douter, n'entreront jamais dans la langue, ils possèdent la vertu de révéler une certaine malléabilité de la langue qui, si cela peut effectivement rendre l'accès au sens difficile, permet de toujours repenser l'évidence de l'expression. Ces «percées novatrices », pour reprendre les termes d'Anne-Marie Houdebine (1985:7) jouent donc un rôle intéressant dans la remise en cause de schèmes de pensées véhiculés par un usage-reflexe, machinal et reposant de la langue. C'est donc bien dans le tourment d'une recherche poétique d'une appréhension sensible du langage que s'élaborent ces structures accidentées, impossibles en langue mais qui peuvent subvertir - au-delà de la forme grammaticale - l'indiscutable et l'indiscuté pour laisser entrevoir un autre état des choses, d'autres possibles.

\section{Possible / impossible et structure argumentale}

Outre l'intégration du complément dans la construction phrastique, c'est parfois l'organisation même de la valence verbale qui est modifiée de façon à donner lieu à des tournures linguistiquement impossibles.

\subsection{Ajout d'un argument}

Ce genre de transformation apparaît à plusieurs reprises :

(6) Je vous entrerai dans ma maison provisoire. ${ }^{10}$

(7) Je suis déjà suicidé. (7') On m'a suicidé, c'est-à-dire. ${ }^{11}$ 
(8) Je parle parce que je veux qu'on me taise, (8') qu'on m'assassine la parole ${ }^{12}$

(9) Tu me suicides, si docilement / (9') Je te mourrai pourtant un jour ${ }^{13}$

(L'étonnante unité des signifiés est apparue de façon tout à fait fortuite lors de notre relevé.)

L'exemple (6) transforme le verbe entrer à structure bivalente (entrer quelque part) en verbe à structure trivalente (entrer quelqu'un quelque part), ajoutant directement un argument sans passer par la diathèse factitive, donc en ne mettant pas le sujet en position d'agent, et l'objet en position de patient. Cette construction court-circuite l'usage normal : «je vous ferai entrer dans ma maison», ce qui provoque une réorganisation de l'expérience, avec un objet (ici [+humain]), et de son récit, puisque cela offre, malgré l'ajout d'un actant, une construction plus « "économique" que les diathèses grammaticales » selon les termes de Krötsch et Oesterreicher (2003).

L'interprétation est semblable pour $\left(9^{\prime}\right)$ : « je te mourrai » pouvant remplacer « je te ferai mourir » ou « je te donnerai la mort ». On a donc une forme qui mélange construction avec pronom datif et construction pronominale (avec l'empreinte de «se mourir » et l'écho au verbe de la première partie du vers «se suicider »), avec un pronom personnel conjoint mais non réfléchi, ce qui provoque, avec l'agrammaticalité, une redéfinition du verbe avec participation active de son sujet. Enfin, une autre lecture possible se superpose, si l'on interprète le pronom personnel comme un datif éthique, avec, dans ce cas, une "invitation directe au destinataire à s'investir affectivement dans l'action décrite » (Riegel, Pellat, Rioul, 2009 : 226). Dans tous les cas, cette variation actancielle crée, là aussi, de l'instabilité dans la langue, et force à repenser les rapports de force dans des actions a priori potentiellement dépourvues d'intentionnalité.

L'ambiguïté d'interprétation du pronom personnel se retrouve en (8'), et tient à l'ajout d'un argument : soit on assassine quelqu'un («on m'assassine »), soit on assassine quelque chose (la parole), mais il est linguistiquement impossible de trouver les deux ensemble. Or c'est bien le cas dans cet exemple, où un objet non prévu dans la rection verbale intervient, et que l'on pourrait lire comme un datif inaliénable, transformant « on assassine ma parole » en " on m'assassine la parole », ce qui organise différemment la construction du verbe, et surtout ce qui fait de «la parole» une part essentielle du sujet, qui en est constitutive, au même titre que les parties du corps. Nous trouvons donc, encore une fois, dans cet exemple, la manifestation d'une créativité linguistique intéressante qui donne à l'expressivité les moyens de reconfigurer certaines unités phrastiques bien ancrées dans la langue.

\subsection{Introduction d'un sujet non-prévu dans la structure du verbe}

Les trois exemples (7') (8) et (9) procèdent également par ajout d'un actant, qui va transformer un verbe essentiellement pronominal en verbe à diathèse passive. Ainsi, la tournure régulière « je me suis suicidé », « tu te suicides », où le sujet et le pronom sont coréférentiels va être infléchie par l'intégration d'un sujet autre, hétérogène dans cette structure, qui va devenir le sujet réel du verbe, transformant le sujet initial en objet du verbe. Cette passivation est très expressive: «on m'a suicidé», le on sujet étant typographiquement mis en relief par un italique accusateur, et ayant un référent vague et global (en l'occurrence il s'agit de la société, qui bannit tous ceux qui ne se conforment pas à ses exigences, comme dans ce titre d'Artaud: Le Suicidé de la société), est ainsi rhématisé dans l'énoncé, et le suicide du sujet est alors relégué au statut de thème, thème effectivement constant dans l'écriture d'Artaud. Cet exemple est d'autant plus intéressant qu'il suit immédiatement un autre fait de langue impossible, en (7), où la disparition du pronom réfléchi donne au participe passé une allure adjectivale. «Je suis déjà suicidé » se lit donc comme une structure attributive, «suicidé » étant interprété comme une propriété caractéristique du sujet. 
L'exemple (9) s'analyse dans les mêmes termes que (7'), à ceci près que le nouveau sujet du verbe est défini. Mais l'interprétation sémantique reste la même : tu me pousses au suicide, tu fais que je me tue, mettant encore une fois l'emphase sur ce sujet autre, non coréférentiel, qui est l'instigateur d'une action réalisée, et subie par celui qui a perdu sa fonction sujet et n'est plus qu'objet.

En (8), nous retrouvons cette présence d'un sujet extérieur et indéfini : « on me tait» au lieu de «je me tais ", avec à nouveau le détournement d'une diathèse factitive : je veux qu'on me fasse taire, qu'on me réduise au silence. La tournure exprime ainsi la nécessité d'un recours extérieur pour réaliser l'action.

Finalement, ces transformations de la valence verbale, en réorganisant l'expression, donnent à lire une nouvelle compréhension du monde, particulièrement frappante dans les exemples (7) et (8) entre autres, où la structure nouvelle avec un sujet supplémentaire lève le voile sur un acteur occulté dans l'usage traditionnel du langage. Ces changements de structures révèlent ainsi la perception ou l'intuition poétique, et va la faire exister dans la langue, puis, éventuellement, dans la parole.

A cet égard, il est intéressant de remarquer que des études en linguistique de corpus - notamment dans les corpus oraux ou tirés de la presse quotidienne - rencontrent des énoncés similaires. Ainsi Krötsch et Oesterreicher (2003) ont-ils relevé par exemple :

Entrer des données (dans l'ordinateur).

La banlieue par ceux qui la bougent.

Comment Chirac a suicidé la droite.

On démissionne le ministre.

La douleur des pères agit les filles.

Oh non, j'ai été divorcé, j'ai été divorcé en quinze jours, on m'a pas demandé mon avis. Je suis revenu d'Afrique, $j$ 'étais divorcé. (Louis-Ferdinand Céline) ${ }^{14}$

Ces exemples récents (à l'exception du dernier cité) ne nous mèneront pas à conclure que les surréalistes ont laissé une empreinte telle que leurs recherches poétiques se retrouvent dans la parole quotidienne, mais ils nous importent en ce qu'ils sont le signe que l'impossible de langue s'est transformé en fait de langue, attesté et grammatisé. L'évolution et le renouvellement de la langue passe donc bien aussi par ces expériences poétiques qui, non seulement disent autrement, en intégrant l'étrange dans la langue, mais aussi peuvent «anticiper sur des formes grammaticales à venir » (Aroui, 1996 : 9-10). C'est bien en ce sens qu'il est possible d'affirmer que le texte littéraire a une fonction instituante dans la langue

\section{Conclusion}

De ces exemples analysés, nous souhaitons retenir le fait que s'ils peuvent actualiser une potentialité de la langue, c'est en allant chercher, au-delà de l'usage ordinaire de la langue, des lieux stratégiques où l'instabilité pourra être introduite de manière fructueuse. Et il n'est pas anodin de remarquer que ces lieux sont toujours situés autour d'un verbe, axe central de la prédication en français, et matrice organisationnelle des propositions. Altérer l'organisation verbale, c'est transformer le cœur même de l'acte de prédication, et ainsi bousculer la façon de délivrer un contenu propositionnel. Face à des énoncés déviants comme ceux exposés, la première lecture consiste sûrement à réduire la part d'anomalie qu'ils présentent en les rapprochant de leurs structures régulières, donc en les comparant à des énoncés qui relèvent incontestablement du possible de langue, en d'autres termes, en les «traduisant» en langage courant. Il y aurait donc une opération de reconnaissance de structures sous-jacentes détournées, et s'il est aisé de les reconnaître, il n'en reste pas moins que leur traitement automatique est contrarié, provoquant, outre la surprise, une véritable « défamiliarisation » telle que l'ont conceptualisée les Formalistes russes. Ce qui se joue, c'est donc bien le changement des représentations véhiculées par le langage, représentations héritées, mobilisées dans chaque acte de parole. Ces structures déviantes, finalement, 
interviennent pour émanciper non pas le langage lui-même, langage toujours dépendant d'un usage, mais bien les sujets-parlants, locuteurs comme poètes, dont la capacité à inventer de nouvelles formes d'expression est infinie. Certes les nouvelles formes créées peuvent s'avérer trop éloignées du mode de signification traditionnel, mais, et cela apparaissait de manière évidente pour les surréalistes, les anomalies ou monstruosités dans la langue provoquent un acte de lecture différent qui génère aussi de la signification. C'est bien l'imagination qui va établir la transition entre tout ce qui est possible et attesté en langue, et ce qui est potentiel, latent, encore impossible mais en gestation, ainsi que cela apparaît sous la plume de Breton :

\begin{abstract}
Je dis que l'imagination, à quoi qu'elle emprunte et - cela pour moi reste à démontrer - si véritablement elle emprunte, n'a pas à s'humilier devant la vie. Il y aura toujours, notamment, entre les idées dites reçues et les idées...qui sait, à faire recevoir, une différence susceptible de rendre l'imagination maîtresse de la situation de l'esprit. (Breton [1932] $1992: 49$ )
\end{abstract}

Le passage du déjà-pensé, déjà-dit à l'expression d'une pensée vive s'inscrit donc pleinement dans le travail sur la langue, travail qui va pouvoir instituer de nouvelles formes, faisant évoluer la langue à mesure que les objets de pensées changent. C'est-à-dire que la langue va s'adapter aux nouvelles propositions si elles résistent à l'usage, donc va augmenter le spectre de ses possibles. Sur ce point, les travaux du philosophe et psychanalyste Castoriadis nous éclairent :

\begin{abstract}
C'est une chose de dire que l'on ne peut choisir un langage dans une liberté absolue, et que chaque langage empiète sur ce qui « est à dire». C'est une autre chose, de croire que l'on est fatalement dominé par le langage et qu'on ne peut jamais dire que ce qu'il vous amène à dire. Nous ne pouvons jamais sortir du langage, mais notre mobilité dans le langage n'a pas de limites et nous permet de tout mettre en question, y compris même le langage et notre rapport à lui. (Castoriadis, 1975 : 176)
\end{abstract}

Explorer cette «mobilité dans le langage » est bien au cœur des recherches poétiques des surréalistes, qui envisagent d'interroger le rapport au langage pour changer nos représentations et ainsi influer sur le monde directement. Ce qui nous intéresse dans cette démarche, c'est bien le fait d'envisager l'usage à la langue non pas en termes de performance accrue, mais bien en termes de nouvelles compétences linguistiques, qui ont éclos au détour de recherches d'une nouvelle façon de dire. Ces recherches ont amené ces poètes à faire un usage extensif d'une grammaire commune, usage extensif qui permet justement de déplacer la limite du possible de langue, ouvrant dans la langue-même un espace nouveau et inédit pour recevoir de nouveaux éléments. Nous trouvons là le point de cristallisation de ces expériences d'émancipation de la pensée par la langue, comme l'exprime Breton

De toute manière nous nous estimerons assez d'avoir contribué à établir l'inanité scandaleuse de ce qui, encore à notre arrivée, se pensait et d'avoir soutenu - ne seraitce que soutenu - qu'il fallait que le pensé succombât enfin sous le pensable. (Breton [1929], $1988: 821$ )

Concevoir les liens de dépendance entre pensée et langage de façon si resserrée explique donc une partie de la production surréaliste qui, montrant que la langue a beaucoup plus de ressources que ce que n'utilise le langage quotidien, emprunte les chemins de traverse de la relation entre la forme de l'expression et le sens, révélant d'autres structurations possibles du discours. Des structurations bien souvent « fluctuantes », qui peuvent retarder ou différer la compréhension de ce qui est dit, obligeant le lecteur à se déplacer dans la suite logique des mots et à les redécouvrir, élargissant considérablement non seulement les moyens expressifs à la disposition du poète, mais aussi l'envergure et la complexité de toute activité de création, de pensée et de lecture. 


\section{Références bibliographiques}

Auroux S. (1991). « Lois, normes et règles », Histoire épistémologie langage, $\mathrm{n}^{0}$ 1, vol. 13, p. 77-107.

Auroux S. (2010). La philosophie du langage, Paris : Que sais-je?

Bonhomme M. (2009). "Mot-valise et remodelage des frontières lexicales », Cahiers de praxématique, vol. 53, p. 99-120.

Breton A. [1927] (1992). Introduction au discours sur le peu de réalité, Oeuvres complètes II, Paris : Gallimard

Breton A. [1929] (1988). Second Manifeste du Surréalisme, Oeuvres complètes I, Paris : Gallimard

Breton A. [1932] (1992). "Il y aura une fois », Le Revolver à cheveux blancs, Oeuvres complètes II, Paris: Gallimard

Breton A. [1955] (2008). Du Surréalisme en ses xuvres vives, Oeuvres complètes IV, Paris : Gallimard

Castoriadis C. (1975). L'Institution imaginaire de la société, Paris : Éd. du Seuil.

Culioli A. (1990). Pour une linguistique de l'énonciation : opérations et représentations, Gap : Ophrys.

Franckel J.-J. et Lebaud D. (1990). Les figures du sujet, Gap : Ophrys.

Houdebine A.M. (1985). "Pour une linguistique synchronique dynamique », La Linguistique, vol. 21 / 1, p. 7-36.

Krötsch M. et Oesterreicher W. (2003). « Dynamique des configurations actancielles » Syntaxe et sémantique, $\mathrm{n}^{\circ} 4$, p. 109-137.

Le Guern M. et Rémi-Giraud S. (1986). Sur le verbe, Lyon : Presses universitaires de Lyon.

Leeman D. (1991). "Hurler de rage, rayonner de bonheur: remarques sur une construction en de ", Langue Française ${ }^{\circ} 91$, pp.80-101.

Martin R. (1978). La Notion de recevabilité en linguistique, Paris : C. Klincksieck.

Milner J.-C. (1989). Introduction à une science du langage, Paris : Éd. du Seuil.

Paulhan J. (1920). «Syntaxe », Proverbe, ${ }^{\circ} 1$, p.1

Picoche J. (1986). Structures sémantiques du lexique français, Paris : Nathan.

Reverdy P. (1918). « Syntaxe », Nord-Sud, n 14, p.3

Riegel M., Pellat J.-C. et Rioul R. (2009). Grammaire méthodique du français, Paris : Presses universitaires de France.

Riffaterre M. (1969). « La métaphore filée dans la poésie surréaliste », Langue française, $\mathrm{n}^{\mathrm{O}}$ 1, vol. 3, p. 46-60.

Riffaterre M. (1979). La production du texte, Paris : Éd. du Seuil (Poétique ; 25), 284 p.

Todorov T. (1966). « Les anomalies sémantiques », Langages, no 1, vol. 1, p. 100-123.

\footnotetext{
${ }^{1}$ Publiée dans Martin 1978

2 Tel que cela a été étudié et explicité notamment par Todorov (1966) - dans son article sur les « anomalies sémantiques ", où les anomalies combinatoires, révélées par l'intuition des sujets-parlants, sont soumises à l'analyse métalinguistique - ou encore par Riffaterre $(1969,1979)$ - avec la théorie de l'agrammaticalité, active dans le processus de signifiance.

${ }^{3}$ Georges Malkine, «Texte Surréaliste », La Révolution Surréaliste, n ${ }^{\circ}$, décembre 1924, p.11

${ }^{4}$ Georges Malkine, «Texte Surréaliste », La Révolution Surréaliste, n ${ }^{\circ}$, juillet 1925, p.10

${ }^{5}$ André Breton, Second Manifeste du Surréalisme, [1929] in CEuvres complètes I, Paris, Gallimard (Bibliothèque de la Pléiade), 1988, 1798 p., éd. Bonnet M., p.781

${ }^{6}$ Georges Malkine, «Texte Surréaliste », La Révolution Surréaliste, n 1 , décembre 1924, p.11

${ }^{7}$ Titre d'un poème de Breton, qu'il emprunte à Desnos

${ }^{8}$ André Breton et Philippe Soupault, Les Champs magnétiques, in Breton, Euvres Complètes, tome I, pp.53-54

${ }^{9} \mathrm{http}: / /$ www.cnrtl.fr/definition/savoir

${ }^{10}$ Georges Malkine, «Texte Surréaliste », La Révolution Surréaliste, ${ }^{\circ}{ }^{\circ}$, décembre 1924, p.11

${ }^{11}$ Antonin Artaud, La Révolution Surréaliste, n², janvier 1925, p.12

${ }^{12}$ Benjamin Péret, Le Gigot sa vie son œuvre, in Euvres complètes, Tome 4, Paris, E. Losfeld, 1969, p.81

${ }^{13}$ Robert Desnos, « Au mocassin du verbe », in, Oeuvres, Paris, Gallimard (Quarto), 1999, 1394 p., p.531

${ }^{14}$ Krötsch M. et Oesterreicher W. (2003). « Dynamique des configurations actancielles » Syntaxe et sémantique, $\mathrm{n}^{\circ} 4$, p. 109-137.
} 